\title{
Kincaid, John (ed.) (2019): A Research Agenda for Federalism Studies
}

\section{Cheltenham/Northampton: Edward Elgar. 232 Seiten. $£ 85$}

\author{
Arthur Benz (iD
}

Online publiziert: 16 . Juli 2020

(C) Der/die Autor(en) 2020

Dem Herausgeber des hier zu besprechenden Sammelbandes, John Kincaid, kommt ohne Zweifel das Verdienst zu, mit eigenen Studien und vor allem mit Sammelbänden die vergleichende Föderalismusforschung gefördert zu haben. Nunmehr liegt sein neuester Band vor, der in der Reihe „Research Agendas“ des Verlags Edward Elgar erschienen ist. Der Verlag will mit dieser Reihe künftige Forschungsthemen in verschiedenen Themenfeldern erschließen, dies durch Beiträge, die sowohl relevant wie visionär sein sollen.

Um eine Forschungsagenda aufzustellen, muss das Forschungsfeld umschrieben werden. Die Abgrenzung des mit Föderalismusstudien bezeichneten Feldes ist schon deswegen schwierig, weil der Begriff Föderalismus unscharf ist. John Kincaid umschreibt ihn einleitend mit „certain key characteristics“ (S. 2), nach denen üblicherweise föderale Systeme definiert werden. Doch die in dem Band behandelten Themen weisen über diesen Begriff eines politischen Systems hinaus. Sie reichen von der Ideengeschichte des Föderalismus, besonderen Forschungsansätzen, Aspekten der Verfassung, Entwicklungen und Herausforderungen bis hin zu Föderationen in den bislang unterbelichteten Kontinenten Asien und Afrika sowie in der EU.

Die in dem Band versammelten Beiträge, die in der Tat aus der Feder von ,leading scholars" stammen und überwiegend von hoher Qualität sind, geben einen komprimierten Überblick über den aktuellen Forschungsstand und künftige Forschungsperspektiven. Sie lassen die unterschiedlichen Zugänge der Fachdisziplinen bzw. Subdisziplinen zur Föderalismusforschung erkennen, zeigen aber auch, wie sich in diesem Gebiet politische Theorie, empirische und vergleichende Politikwissenschaft, Rechtswissenschaft und Finanzwissenschaft wechselseitig stimulieren. Das Spektrum der theoretischen bzw. analytischen Zugänge, die in den einzelnen Kapi-

\footnotetext{
A. Benz ( $\square)$

Institut für Politikwissenschaft, Technische Universität Darmstadt, Darmstadt, Deutschland E-Mail: arthur.benz@tu-darmstadt.de
} 
teln zum Ausdruck kommen, umfasst normative, akteurs-, institutionen- und gesellschaftszentrierte Ansätze und wird ergänzt durch Area Studies.

Die Themenauswahl deckt wichtige Forschungsfelder ab, bleibt aber notwendigerweise selektiv. Dabei kann man über die Gewichtung geteilter Meinung sein. Aspekte der Verfassung erscheinen mir mit drei, wenngleich interessanten, Beiträgen überbetont. Das Spannungsverhältnis zwischen Föderalismus und gesellschaftlicher Diversität wird in vier Beiträgen behandelt, wobei André Lecours eine vielversprechende Forschungsperspektive aufzeigt. Zentralisierung und Dezentralisierung sowie „shared rule“, denen zwei Kapitel gewidmet sind, sind traditionelle Kategorien, die erst dann innovative Forschung stimulieren, wenn die vielfältigen Muster von ,intergovernmental relations" und ihre Effekte auf Policies oder auch Machtdynamiken berücksichtigt werden, die in dem Band zu kurz kommen.

Unterbelichtet bleiben etwa Parteien und Parteiensysteme in Föderationen, die in letzter Zeit intensiv untersucht wurden, Interessengruppen und Zivilgesellschaft, die in diesem Zusammenhang weniger erforscht sind, die besonderen Effekte von Wahlen und Abstimmungen in Bundesstaaten, der Zusammenhang von Föderalismus und Formen von Demokratie oder Autokratie (den allerdings Harihar Bhattacharyya für das Verständnis des Föderalismus in asiatischen Staaten herausgestellt) sowie historische Ansätze, die der Föderalismusforschung in Nordamerika jüngst einen neuen Aufschwung verliehen haben. Der Einleitung ist zu entnehmen, dass der Herausgeber solche Forschungsarbeiten nicht der Föderalismusforschung, sondern anderen Bereichen der Politikwissenschaft zuordnet, während die Kapitel des vorliegenden Sammelbandes Kernbereiche der Föderalismusforschung erschließen sollen. Aber gerade Forschung an Schnittstellen der Fachgebiete hat sich als besonders fruchtbar erwiesen und der Band belegt dies auch.

Die methodischen, konzeptionellen und theoretischen Herausforderungen der Föderalismusforschung kann man dem Beitrag von Alan Fenna entnehmen, der die Frage stellt, was vom „comparative federalism“ zu erhoffen ist. John Kincaid skizziert in seiner Einleitung die Breite des Forschungsgebiets, die der Band dennoch abzudecken versucht. Am Ende dieses Beitrags plädiert er dafür, Föderalismus als Ganzes - ,the whole of federalism“ (S. 13) - in den Blick zu nehmen, um nicht, „like the blind man and the elephant, fail to see the whole for the part" (S. 13). Dem ist grundsätzlich zuzustimmen. Doch wie der Band zeigt, gibt es zum Föderalismus unterschiedliche Sichtweisen des Ganzen und auf das Ganze.

Wer sich für die Föderalismusforschung interessiert, gewinnt durch die Lektüre dieses Bandes einen guten Überblick über das Forschungsfeld. Es wäre zu wünschen, dass die Anregungen für die künftige Forschung, die alle Kapitel enthalten, aufgegriffen werden. Forschungslücken und offene Fragen gibt es genug.

Funding Open Access funding provided by Projekt DEAL.

Open Access Dieser Artikel wird unter der Creative Commons Namensnennung 4.0 International Lizenz veröffentlicht, welche die Nutzung, Vervielfältigung, Bearbeitung, Verbreitung und Wiedergabe in jeglichem Medium und Format erlaubt, sofern Sie den/die ursprünglichen Autor(en) und die Quelle ordnungsgemäß nennen, einen Link zur Creative Commons Lizenz beifügen und angeben, ob Änderungen vorgenommen wurden. 
Die in diesem Artikel enthaltenen Bilder und sonstiges Drittmaterial unterliegen ebenfalls der genannten Creative Commons Lizenz, sofern sich aus der Abbildungslegende nichts anderes ergibt. Sofern das betreffende Material nicht unter der genannten Creative Commons Lizenz steht und die betreffende Handlung nicht nach gesetzlichen Vorschriften erlaubt ist, ist für die oben aufgeführten Weiterverwendungen des Materials die Einwilligung des jeweiligen Rechteinhabers einzuholen.

Weitere Details zur Lizenz entnehmen Sie bitte der Lizenzinformation auf http://creativecommons.org/ licenses/by/4.0/deed.de. 